\title{
Modular Approach based Backbone Construction Using STP with CDS
}

\author{
Rachita Nagpal $^{\mathrm{a} *}$, Roopali Garg ${ }^{\mathrm{b}}$ \\ ${ }^{a}$ Research Scholar, Department of I.T, U.I.E.T, Panjab University, Chandigarh, 160014, India \\ ${ }^{b}$ Assistant Professor, Department of I.T, U.I.E.T, Panjab University, Chandigarh, 160014, India
}

\begin{abstract}
In a dense environment, wireless sensor network (WSN) requires more energy to work in an effective and efficient manner. Hence, energy conservation is the main objective. In the paper, we have proposed a methodology to construct a backbone using modular antennas in combination with spanning tree protocol (STP), graph sampling, and Connecting Dominating Set (CDS) strategy. The backbone construction is based upon the modular antenna based WSNs, where the dominating sets can avoid the intermediate connection in order to reduce the hop count and energy consumption. The dominating sets have been connected using the modular transmission range of the wireless sensor networks to construct the backbone. The dominating set selection procedure to construct the WSN backbone is based upon the degree of connections of the nodes, which enables the locally centralized behavior of the connected dominating sets. The proposed methodology has been proved effective resulting in the construction of an energy efficient backbone.
\end{abstract}

Index Terms: Non-Probabilistic Spanning Tree, Connecting Dominating Set (CDS), Graph Sampling, Dominating Sets, Spanning tree Protocol (STP).

(C) 2015 Published by MECS Publisher. Selection and/or peer review under responsibility of the Research Association of Modern Education and Computer Science.

\section{Introduction}

Wireless Multimedia Sensor Networks (WMSN) has become a medium to transfer data such as images, audio and video other than scalar data. Sensor networks consist of battery operated devices that consume energy to accomplish their tasks. The number of sensor nodes in the WSN network varies from a few sensor nodes to large number of sensor nodes depending upon the application for which it is designed.

For continual working of the sensor device, energy should be conserved. Many efforts have been done to conserve energy in WSN networks but still energy cannot be conserved in an efficient manner.

When the WSN network is initialised, the nodes enter into active state. Some of the nodes may also enter

* Corresponding author. Tel.: +91-9592206069

E-mail address: rachitanagpal1891@gmail.com 
into idle state. At this point of time, the node of the WSN network are active but neither sends nor receives the packet. In idle state, there are many elements of the sender and receiver connections that cannot be turned off to conserve energy. This energy consumption is negotiable where small number of sensor nodes are deployed but unavoidable in dense WSNs.

Analysing energy consumption in dense topology is difficult due to the presence of large number of nodes and complexity of the network. This problem is solved through the implementation of graph sampling. Graph sampling is done in order to reduce the complexity of the network. In graph sampling process, sampling algorithms are applied to obtain a sample network from the original network. The resultant sample network is used to represent the original network with less number of nodes as compared to the original network.

The previous researchers show that the energy of the network is improved by applying graph sampling and connecting dominating set in sequence. Here graph sampling reduces the complexity of the network and connecting dominating sets is used to construct a backbone.

We proposed to build a backbone framework using modular antennas along with spanning tree algorithm, graph sampling and CDS strategy. The proposed methodology when simulated in MATLAB has proved to be effective and has helped in the construction of an energy efficient backbone framework.

The rest of the paper is divided into various sections as follows: Section II discusses the work from which the proposed model is inspired; Section III presents the process used to obtain an energy efficient backbone; section IV shows the results obtained. Finally, section V draws the conclusion and future scope.

\section{Motivation}

Many research has been done to conserve energy. Deepti Garg et al., 2012 have proposed an angled-LEACH protocol [1], in which energy efficiency is achieved by reducing traffic during communication between the nodes. Roopali Garg et al., 2013 have focused on enhanced version of the LEACH protocol that is used to save energy and had also helped in increasing the lifetime of the network [2].

Kayhan Erciyes et al., 2008 implemented a spanning tree protocol to form clusters in the hierarchical structure with the sink node. Then this distributed approach was improvised to follow the shortest path to communicate with the parent node [3].

Partha Basuchowdhuri et al., 2014 discussed about community structure detection in social networks that has been done using spanning tree protocol [4]. The objective has been achieved in three steps. The first step involves the extraction of the un-weighted graph from the weighted graph. The second step involves the calculation of maximal spanning tree and the last step includes the formation of sub-graphs with the nodes present in clusters.

Paola Flocchini et al., 2012 had discussed problems in social networks, such as node replacement problem and edge replacement problem [5] and had inferred that these problems are solved by extracting a spanning tree from the original network following minimum cost. Formation of spanning tree provides a better-connected graph without loops.

Xiaohua Xiong et al., 2014 described a competitive decision algorithm [6] that has been used for effective energy utilization which has helped in increasing the working capability of the network. This is achieved by dividing the load equally among the WSN network.

Sijun Ren et al., 2014 constructed a backbone using Connected Dominating Set (CDS) strategy [7]. This strategy helps building an energy efficient backbone that divides the load of the network and increases the lifetime of the network. The backbone is constructed based on the number of degrees a node in the network possesses.

Jure Leskovec et al., 2006 discussed various graph sampling algorithms in [8]. In this paper, the authors have described various graph sampling algorithms such as random walk, Breadth-first search etc. Various spatial and temporal properties are also described that are further used to compare the sampled graph with the original graph.

Ravindara Bhatt et al., 2014 have constructed a backbone using graph sampling and CDS strategy in [9]. 
A 
backbone for a complex WSN network has been constructed that has increased the efficiency and lifetime of the network.

The various concepts related to the construction of backbone has been discussed by Hongwei Du et al. 2013 in [10] and by Yu Xiang et al., 2012 in [11]. Ci Song et al., 2005 in [12], described an enhanced version of MAC protocol, which was used to optimize wireless sensor networks in terms of performance and fairness. A link adaptation technique is used to improve the utilization of energy in an efficient way. In this technique, a variable-sized frame size is used for data transmission and to reduce the computational complexity a filter is used to estimate the optimal size of the frame that helps in improving performance and throughput leading to low energy consumption.

N. Tezcan et al., 2006 in [13], discussed a scheduling scheme used to enhance energy efficiency. This scheduling algorithm was based on a two-tier architecture. Using this scheme the sensor nodes were allowed to enter into sleep mode. This was done by selecting a set of nodes for communication and the non-selected nodes are put into sleep mode. The sensor nodes may be put into either of the two sleep modes: continuous sleep and periodic sleep. This has helped in restraining the nodes from entering into idle state hence, improving energy efficiency.

Hoang Duc Chinh et al., 2012 in [14], represented how the power consumed by the WSN network to transfer data was taken under consideration. After analysing the power consumed for data transmission, a sensor with the same power configuration was used along with the time scheduling technique for receiving data. The results show that this technique has helped in increasing the lifetime of a sensor node by $32 \%$.

P. Glatz et al., 2011 in [15], described a novel framework is developed by combining energy harvesting routing technique with duty cycling for conserving the bandwidth and the energy of the WSN network. This combined technique is then used with network coding rules and we inferences depict that the energy conservation is improved from $13 \%$ to $50 \%$.

\section{Experimental Design}

The algorithm is designed for the purpose of backbone construction for the dense WSNs. The model aims at the construction of energy efficient WSN backbone with the minimum possible number of nodes as the backbone members (called dominating sets). The dominating sets are the nodes with the highest degree of connections. There are such WSN nodes which can adjust their transmission range according to the availability of their neighbours or according to the node count based threshold. The threshold value will decide the transmission radius of the WSN nodes. The modular antennas are being used in such nodes, which are capable of transmitting the signal within the variable ranges. The modular antennas are built of numerous modules, which are switched on or off according to the requirement. The model uses such modular antennas to increase the range of the dominating sets in order to connect the dominating sets. The connected dominating sets construct the backbone architecture in the WSNs. The newly constructed backbone will be capable of maximizing the lifetime of the WSNs by enabling an energy efficient and effective data delivery system. Spanning tree protocol, graph sampling and CDS technique is implemented to construct an energy efficient backbone framework. Spanning tree protocol is used to increase the connectivity of the network, graph sampling is used to reduce the complexity of the network and the CDS technique is used to build the backbone framework for the WSN. The model works in the following steps:

1. Non-probabilistic spanning tree

2. Connecting dominating sets

3. Graph sampling for connection correction

4. Spanning tree to prune the redundant connections 


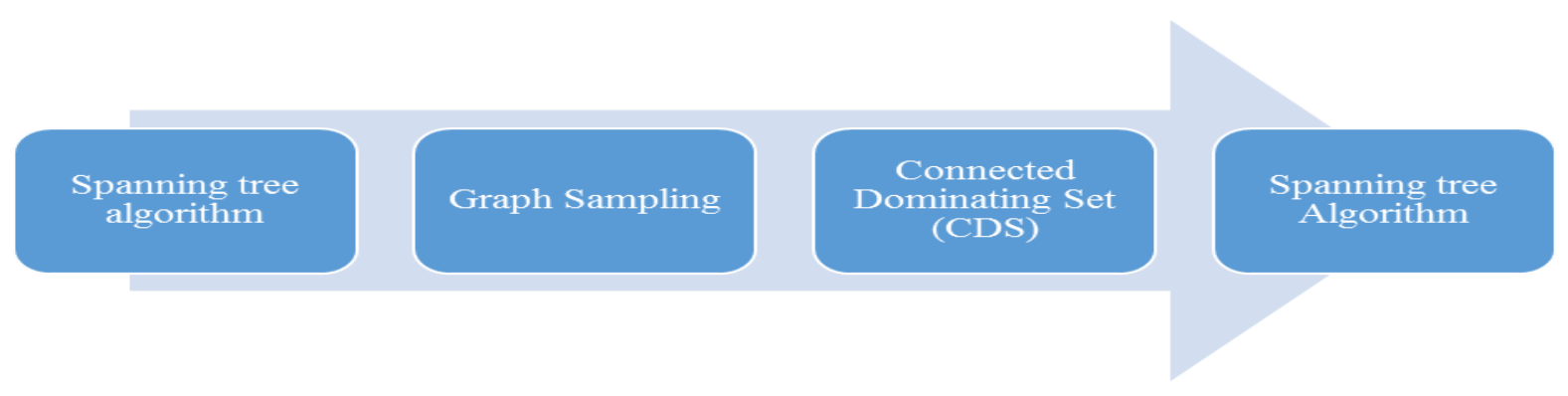

Fig. 1. Methodology

\subsection{Non-Probabilistic Spanning Tree}

The non-probabilistic spanning tree is the mechanisms which does not account for any probability of the connections, but checks the degree of connections to connect the nodes at first level. At this step, the spanning tree has been programmed to check for the redundant links with the lower degrees than other alternative contenders. Also, it blocks the alternative links where the probability of routing loops exist. The nonprobabilistic spanning is inspired by the idea of initial connectivity with no existence of the redundant links.

\subsection{Connecting Dominating Sets}

After the initial connectivity using the non-probabilistic spanning tree, the dominating sets are evaluated and the nodes with the higher order (of connections) are connected to each other using the connected dominating sets. The connected dominating sets algorithm is the method, which is used to create the backbone by connecting the nodes with the higher degree of regional connections to construct the central backbone of the network architecture. The purpose of the connected dominating sets is to connect the nodes by using the dynamic transmission range scheme. The dynamic transmission range is adjusted according to the distance of the neighbouring dominated sets.

\subsection{Graph Sampling for Correcting the Connection}

Then it comes to the graph sampling. The graph sampling is the scheme where the connection weights are corrected by calculating the highest probability of fitness of all the nodes in the sampling window. The graph sampling is the process of correcting the connection by deleting the extra connections or adding the new connections, or both, according to the requirements found during the node and link domination probability calculation.

\subsection{Spanning Tree for Pruning Redundant Connections}

The probabilistic controlled spanning tree would be applied on the topology after completion of the graph sampling to prune the redundant connections between the dominated sets. When the dominated sets are connected using the extended transmission range, the routed links between them are not checked due the vulnerability of the CDS algorithm to take on the redundancy issues. Hence, the redundancy issues are taken care by the spanning tree which is considered the best algorithm for such purpose.

\section{Simulated Results}

The results are simulated using MATLAB. The original network consisting of 120 nodes are shown in Fig 2. 
The position of the nodes are assigned using static values. Rachita Nagpal et al., 2015 have implemented nonprobabilistic spanning tree approach [16] on the original network in Fig 2. According to this approach, the WSN nodes first start with the initialization process in which the nodes of the network enter into active state. Then, the nodes collect the information of their neighbouring nodes and complete their routing tables. The nodes that fall within the transmission are connected.

The spanning tree protocol is used to avoid redundant links and loops and also the protocol is used to obtain a connected tree structure shown in Fig 3. CDS strategy is then applied on the tree structure obtained in Fig 3 to obtain the backbone nodes that will help in performing routing function. The dominating nodes obtained in red color are the nodes obtained by implementing spanning tree and CDS strategy in a sequence. This is presented in Fig 4. Graph sampling is implemented on Fig 4 to obtain the sampled graph of the original network. Graph sampling is implemented to reduce the complexity of the graph. Graph sampling is performed on area basis i.e. a block size equivalent to the transmission radius is fixed. The block size is taken as 25 to perform graph sampling. This graph sampling technique also corrects the connectivity between the nodes and the dominating nodes. The corrected connections obtained after graph sampling is shown in Fig 5 and the dominating nodes are represented in blue color.

Lastly, spanning tree is implemented to prune the redundant connections between the dominating sets. The network obtained with pruned redundant connections is shown in Fig 6. To obtain the backbone framework, CDS technique is implemented. The resultant backbone obtained is shown in Fig 6. As seen in the figure, more randomness is seen while transferring the data through the backbone framework. This randomness problem is solved using the concept of modular antennas which helps in building a backbone framework with les number of hop counts. The backbone obtained with fewer hop counts is shown in Fig 7 and so is energy efficient.

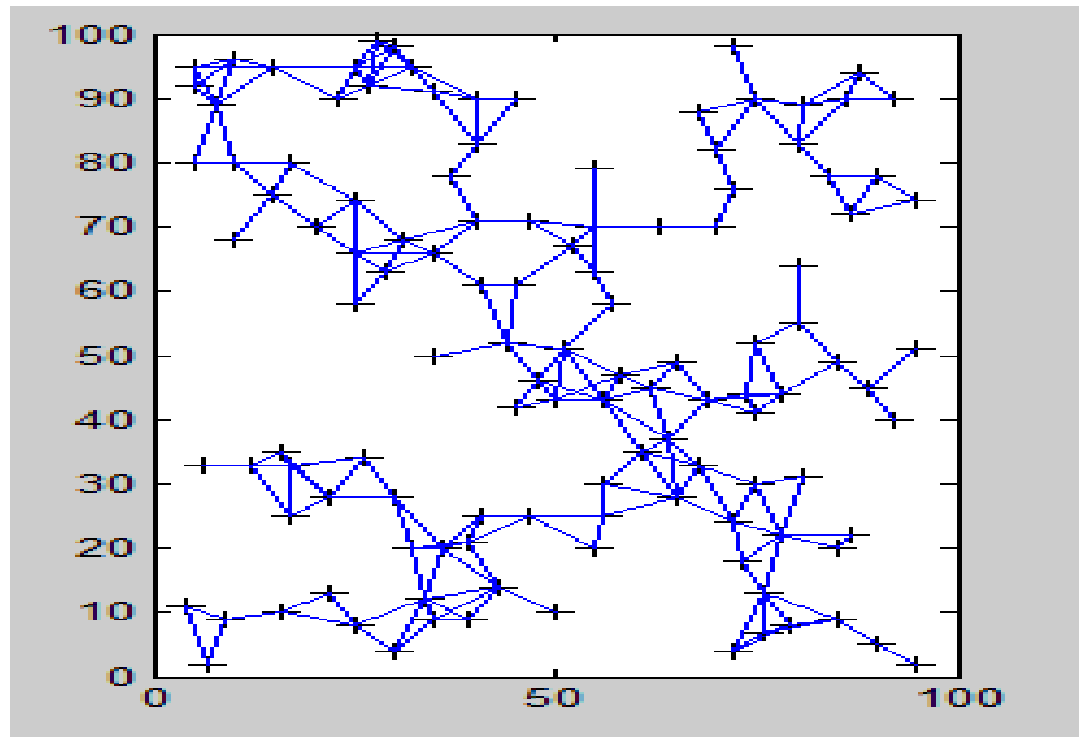

Fig. 2. Original Network [16] 


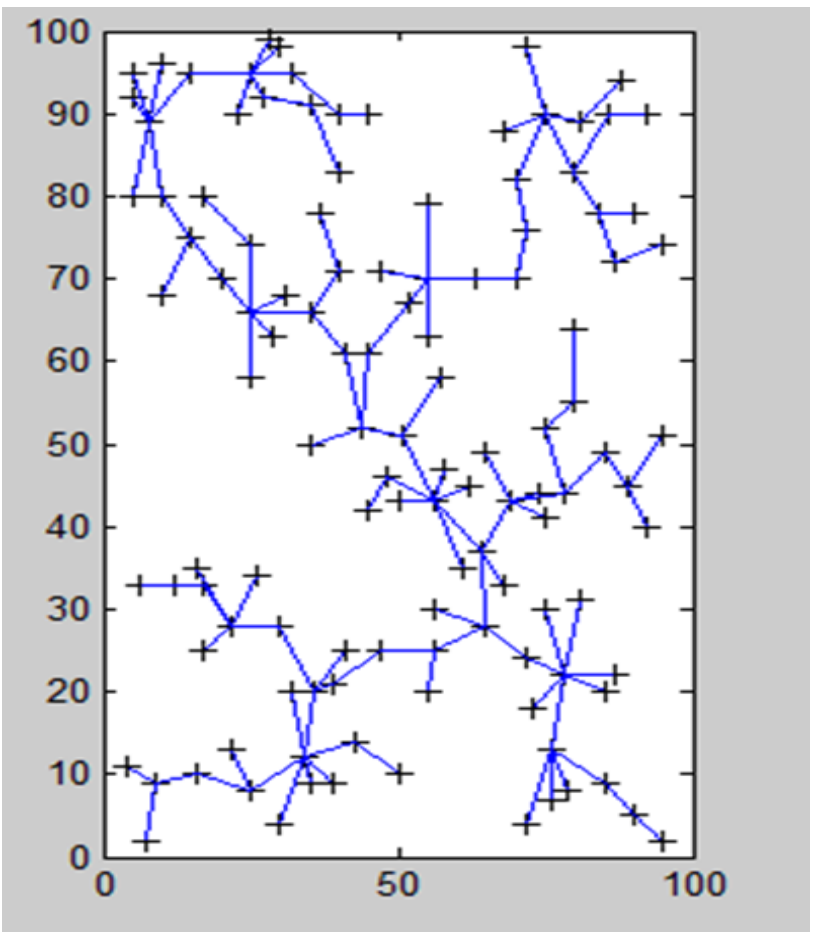

Fig. 3. Spanning Tree Obtained After Implementing Non-Probabilistic Approach [16]

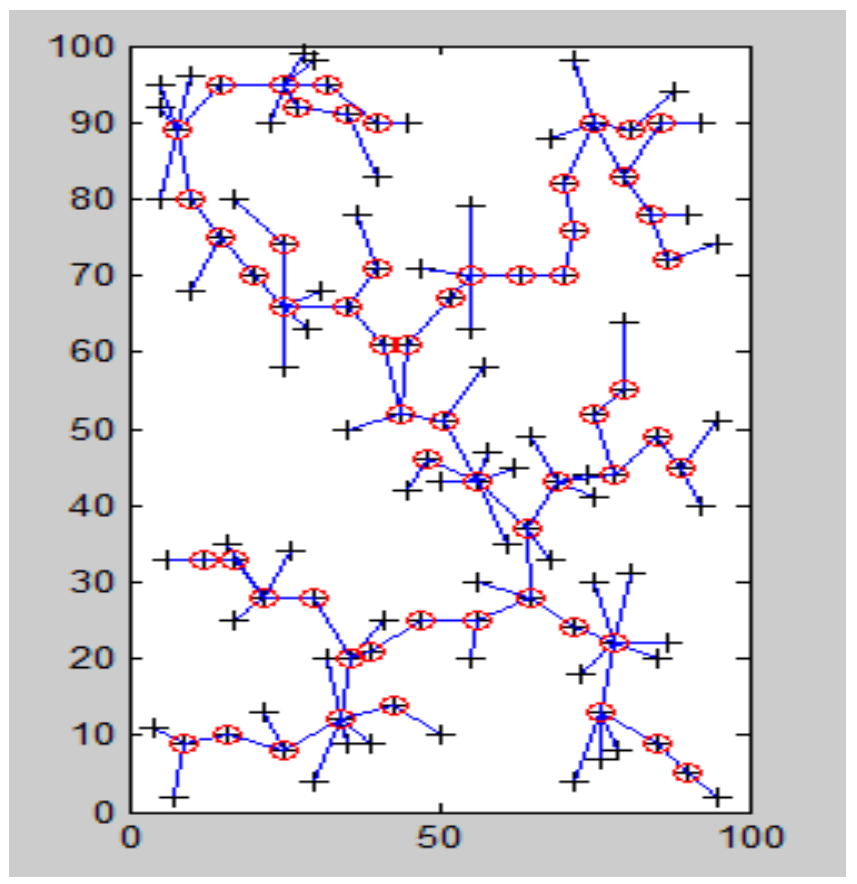

Fig. 4. Spanning Tree with Backbone Nodes (Without Graph Sampling) [16] 


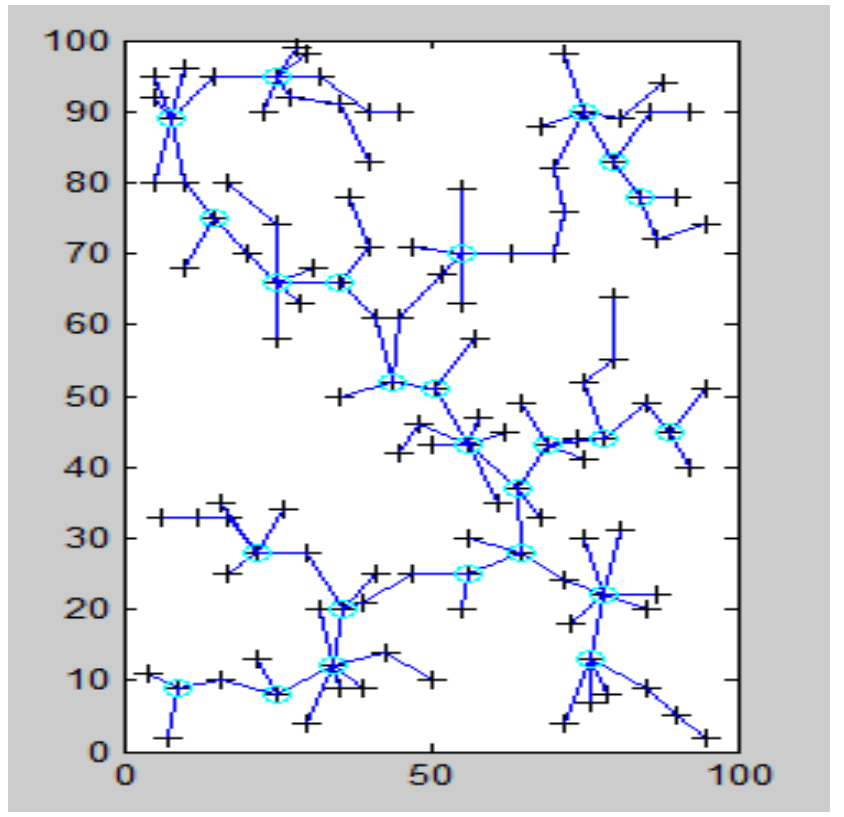

Fig. 5. Backbone Nodes Obtained After Graph Sampling

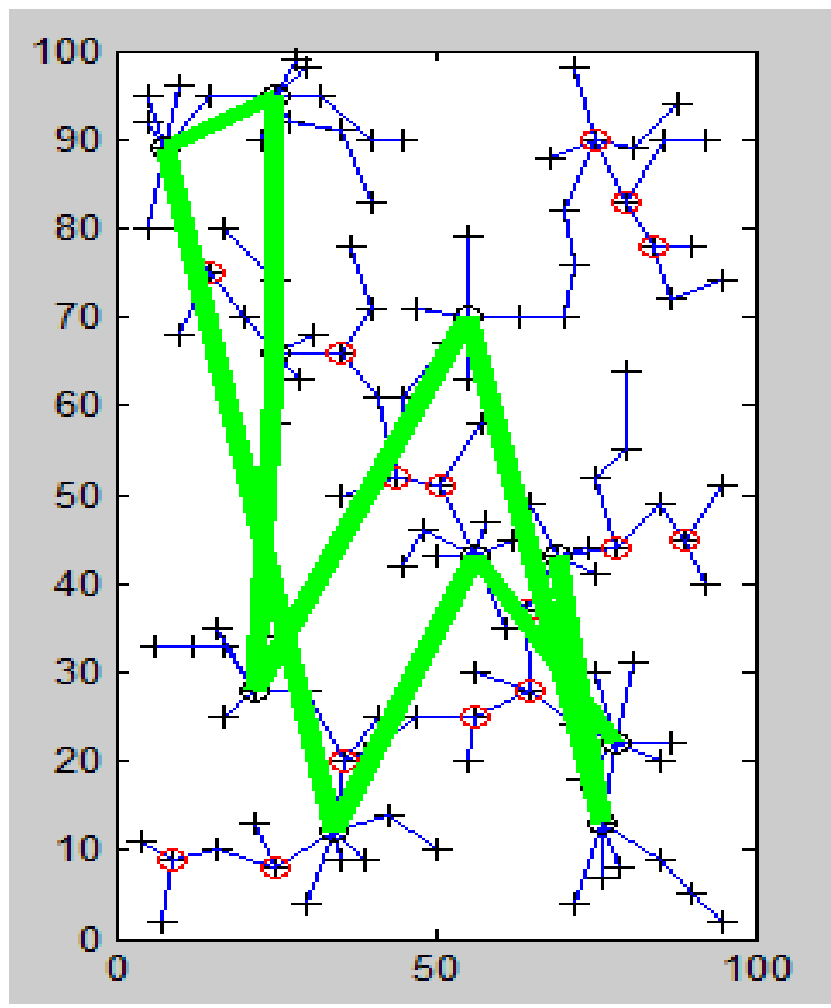

Fig. 6. Backbone Construction (without using Modular antennas) 


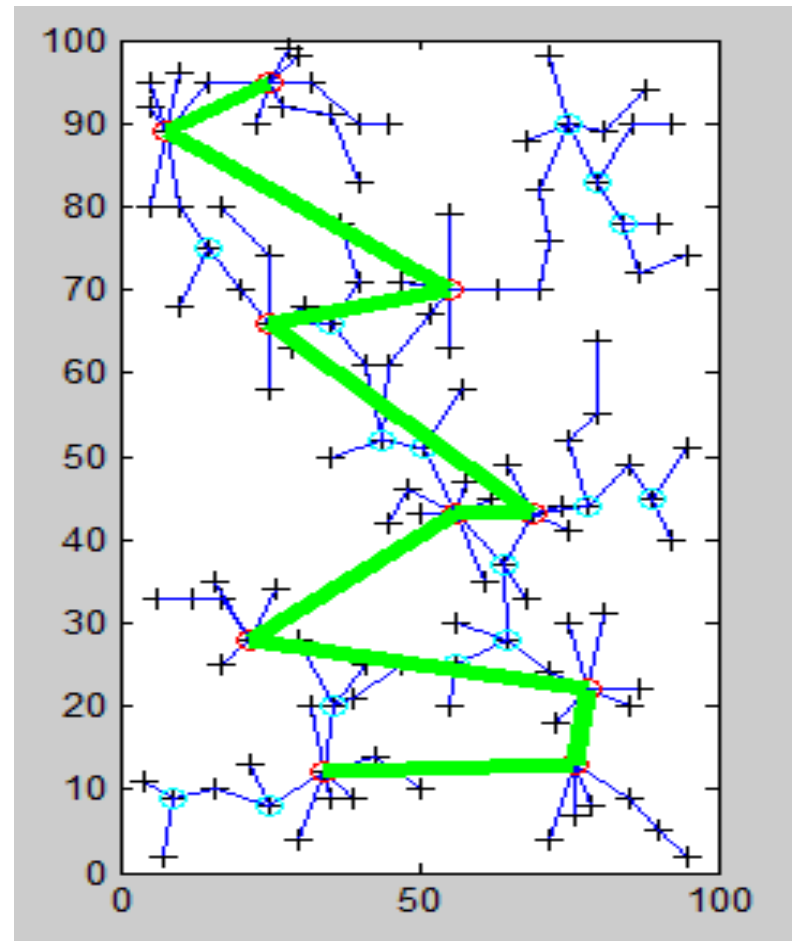

Fig. 7. Backbone Framework (Using Modular Antennas)

\section{Conclusion and Future Scope}

The above-defined methodology helps in the construction of backbone using spanning tree protocol, graph sampling and CDS strategy. The backbone framework that is obtained using the above process helps in the utilization of energy in an efficient way and also helps in increasing the lifetime of the network. The spanning tree implemented in this paper helps in providing a better connectivity to the network. This connected network is then passed to CDS to construct backbone. The graph sampling corrects the connectivity and establishes or deletes the connections that fall out of the sampling window. Lastly, implementation of the spanning tree in the end helps in pruning of the connections.

Using the concept of modular antennas helps in controlling the transmission range. Also, it avoids the intermediate connection between dominating sets which further reduces the hop count and thus increases the energy of the network.

In future, the model will be enhanced for backbone security and also for providing a higher order of flexibility and stability. Also, the model can be enhanced using the different techniques or combination to construct the backbone of the dense WSNs in an efficient way.

\section{Acknowledgements}

I would like to thank my co-author Mrs. Roopali Garg for providing me with her valuable guidance in this field. Her continuous and valuable support has been of great help in completing the research work. 


\section{References}

[1] D. Garg and R Garg, “Angled-LEACH in Wireless Sensor Networks", International Journal of Advances In Computing And information Technology, 2012

[2] R Garg and D Gupta, " Improving the Network Lifetime in WSN through Enhanced LEACH " , International Journal of Computer Applications, 2013

[3] K. Erciyes, D. Ozsoyeller and O. Dagdeviren, "Distributed algorithms to form cluster based spanning trees in wireless sensor networks." In Computational Science-ICCS 2008, pp. 519-528. Springer Berlin Heidelberg, 2008.

[4] P. Basuchowdhuri, S. Anand, D. R. Srivastava, K. Mishra and S. K. Saha, "Detection of Communities in Social Networks Using Spanning Tree" Advanced Computing, Networking and Informatics, Springer, 2014, pp. 589-597.

[5] P. Flocchini, T.M. Enriquez, L. Pagli, G. Prencipe and N. Santoro, "Distributed Minimum Spanning Tree Maintenance forTransient Node Failures", Computers, IEEE, 2012, pp. 408 - 414.

[6] X. Xiong and A. Ning, "Competitive decision algorithm for constructing maximum lifetime spanning tree in wireless sensor networks", Computer Science \& Education (ICCSE), IEEE, 2014, pp. 1014-1019.

[7] S. Ren, P. Yi, D. Hong, Y. Wu and T. Zhu, "Distributed Construction of connected Dominating Sets Optimized by Minimum-Weight Spanning Tree in WirelessAd-Hoc Sensor Networks", Computational Science and Engineering (CSE), IEEE, 2014, pp. 901-908.

[8] J. Leskovec and C. Faloutsos, "Sampling from Large Graphs," ACM, 2006.

[9] R. Bhatt and R. Datta, "Utilizing graph sampling and connected dominating set for backbone construction in wireless multimedia sensor networks," in Communications (NCC), 2014 Twentieth National Conference on. IEEE, 2014, pp. 1-6.

[10] H. Du, W. Wu, Q. Ye, D. Li, W. Lee and X. Xu. "CDS-based virtual backbone construction with guaranteed routing cost in wireless sensor networks." Parallel and Distributed Systems, IEEE, 2013, pp. 652-661.

[11] Y. Xiang, X. Liu, Y. Tang, J. Xiao and J Zhang. "A distributed algorithm for virtual backbone construction with cellular structure in WSNs." International Journal of Distributed Sensor Networks, 2012.

[12] Song Ci; Sharif, H.; Nuli, K., "Study of an adaptive frame size predictor to enhance energy conservation in wireless sensor networks," Selected Areas in Communications, IEEE, Volume: 23, Issue: 2, pp. 283 $292,2005$.

[13] Tezcan, N.; Wenye Wang, "TTS: A Two-Tiered Scheduling Algorithm for Effective Energy Conservation in Wireless Sensor Networks," Communications ICC '06, IEEE, volume 7, pp 3359 - 3364, 2006.

[14] Hoang Duc Chinh; Panda, S.K., "Real-time power configuration for energy conservation in wireless sensor networks," Communication Systems (ICCS), IEEE, pp. 152 - 156, 2012.

[15] Glatz, P.M.; Hörmann, L.B.; Steger, C.; Weiss, R., "Opportunistic Network Coding for Energy Conservation in Wireless Sensor Networks," Communication Networks and Services Research Conference (CNSR), IEEE, pp. 239 - 246, 2011.

[16] R. Nagpal and R. Garg, "WSN Backbone Formation Using Non-Probabilistic Spanning Tree Algorithm" International Conference on Communication, Computing and Power Technologies, 2015, pp. 749-756, in press. 


\section{Authors' Profiles}
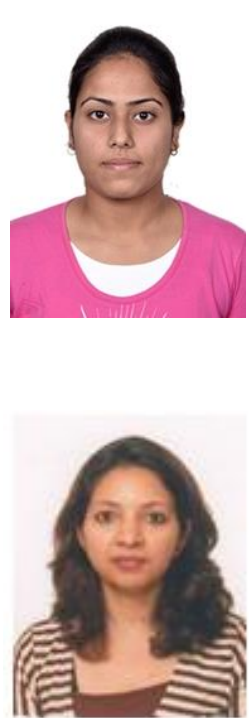

Rachita Nagpal, a post graduate student of Information Technology, is undergoing a mandatory research in the innovative computing technologies. She is pursuing her master's degree from U.I.E.T, Panjab University and has pursued her bachelor's degree from Rayat and Bahra College of Engineering and Biotechnology for women. There are two review and two research papers to her credit which have been published in international journals and have been presented in reputed international conferences. She holds a strong interest in the area of Wireless Sensor Networks and is working strongly towards building the stable backbone for delivering the heavier amounts of data.

Roopali Garg, is working as Assistant Professor at department of Information Technology Engineering at UIET, Panjab University, Chandigarh. She has an experience of 12 years in academics. She has done M. tech in Electronics and B. Tech in Electronics \& Electrical Communication from Punjab Engineering College. She has been awarded Administrator's Gold medal by Chandigarh Administration in 2000 for her supreme performance in curricular, co- curricular and extra- curricular activities. There are more than thirty research papers to her credit which have been published in good indexed international journals and have been presented in reputed international conferences. Her focused research area is Wireless communication and has guided more than a dozen M. thesis in this area.

How to cite this paper: Rachita Nagpal, Roopali Garg,"Modular Approach based Backbone Construction Using STP with CDS", IJEM, vol.5, no.3, pp.1-10, 2015.DOI: 10.5815/ijem.2015.03.01 